\title{
Aislación relativa comparada con absoluta en restauraciones de resina compuesta en pacientes con dentición permanente.
}

\section{Relative isolation versus rubber dam isolation for composite resin restorations in patients with permanent dentition.}

\author{
Macarena Muñoz ${ }^{1,2}$, Nicolás Est \\ 1. Escuela de Odontología, Facultad de Medicina, \\ Pontificia Universidad Católica de Chile, Santiago, \\ Chile \\ 2. Proyecto Epistemonikos, Santiago, Chile \\ 3. Centro Evidencia UC, Facultad de Medicina, \\ Pontificia Universidad Católica de Chile, Santiago, \\ Chile \\ * Correspondencia Autor: María Francisca Vivanco \\ | Dirección: Centro Evidencia UC, Pontificia \\ Universidad Católica de Chile, Diagonal Paraguay \\ 476, Santiago, Chile | E-mail: mvivancot@uc.cl
}

\begin{abstract}
RESUMEN
Introducción: La técnica de restauración con resina compuesta requiere un adecuado control de la humedad durante el procedimiento. Para ello, puede realizarse aislación relativa (con tórulas de algodón y eyector de saliva) o aislación absoluta con goma dique de la o las piezas dentarias. Sin embargo, no está claro cuál de estos protocolos sería más efectivo. Métodos: Realizamos una búsqueda en Epistemonikos, la mayor base de datos de revisiones sistemáticas en salud, la cual es mantenida mediante el cribado de múltiples fuentes de información, incluyendo MEDLINE, EMBASE, Cochrane, entre otras. Extrajimos los datos desde las revisiones identificadas, analizamos los datos de los estudios primarios, realizamos un metaanálisis y preparamos una tabla de resumen de los resultados utilizando el método GRADE. Resultados y conclusiones: Identificamos tres revisiones sistemáticas que en conjunto incluyeron dos estudios primarios, todos correspondientes a ensayos aleatorizados. Concluimos que realizar aislación relativa comparado con aislación absoluta podría aumentar el riesgo de falla de restauración a los 6 meses, pero la certeza de la evidencia es baja. No es posible establecer con claridad si la aislación relativa disminuye el riesgo de falla de la restauración a 10 años, debido a que la certeza de la evidencia existente ha sido evaluada como muy baja. No se encontraron estudios que evaluaran los desenlaces caries secundaria, sensibilidad postoperatoria y tinción.
\end{abstract}

\section{PALABRAS CLAVE}

Restauración; Resina compuesta; Aislación relativa; Aislación absoluta; Aislación con goma dique; Epistemonikos;GRADE.

\section{ABSTRACT}

Introduction: Resin composite restorations technique requires effective control of moisture during the procedure. Isolation and moisture control can be performed by relative isolation (use of cotton rolls combined with aspiration by saliva ejector) or rubber dam isolation. However, there is uncertainty regarding which one of these protocols would be most beneficial. Methods: We searched in Epistemonikos, the largest database of systematic reviews in health, which is maintained by screening multiple information sources, including MEDLINE, EMBASE, Cochrane, among others. We extracted data from the systematic reviews, reanalyzed data of primary studies, conducted a meta-analysis and generated a summary of findings table using the GRADE approach. Results and conclusions: We identified three systematic reviews including two studies overall, of which all were randomized trials. We conclude that relative isolation may increase the risk of failure of the restorations at 6 months. We are uncertain whether relative reduces the risk of failure of the restorations at 10 years as the certainty of the evidence has been assessed as very low. No studies were found that looked at secondary caries, post-operative sensitivity or stained margins.

\section{KEY WORDS}

Restorations; Resin composite; relative isolation; rubber dam isolation; Epistemonikos; GRADE.

Int. J. Inter. Dent Vol. 14(1); 79-82, 2021. 


\section{PROBLEMA}

La resina compuesta es un material restaurador cuyo uso se ha masificado en la operatoria directa actual, siendo una de sus ventajas la estética que ofrece. Pese a su gran aceptación, estudios previos han demostrado que el reemplazo de restauraciones de resina compuesta corresponde entre el 50 al $78 \%$ de la actividad en la práctica clínica. Dentro de los factores que influyen en la longevidad de las restauraciones, encontramos tipo de material, técnica y destreza del operador ${ }^{(1)}$.

La técnica de restauración con resina compuesta se caracteriza por ser muy sensible al contacto con fluidos orales como saliva, sangre y líquido crevicular, por lo cual es necesario realizar un adecuado control

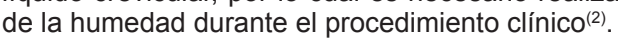

En odontología, la aislación corresponde a la separación de uno o varios dientes de los tejidos orales y la saliva, para mejorar el acceso, la visibilidad y controlar la contaminación por humedad mientras se realizan procedimientos dentales ${ }^{(2,3)}$.

Existen dos técnicas de aislación; la absoluta y la relativa. La aislación absoluta, realizada con goma dique, logra una separación casi en su totalidad del medio oral, disminuyendo el riesgo de aspiración de instrumentales, ayuda a tener una mejor visión y acceso al tratante. Sin embargo, dentro de sus desventajas destaca la incomodidad que produce en el paciente ${ }^{(3)}$. Por otro lado, la aislación relativa es aquella en donde se controla la humedad por medio de tórulas de algodón. Esta aislación es menos invasiva para el paciente pero es necesario realizar un recambio constante de las tórulas durante todo el procedimiento. También presenta un menor control de los fluidos orales en comparación con la aislación absoluta ${ }^{(3)}$

Pese a las ventajas y desventajas conocidas de ambas técnicas, aún existe incertidumbre sobre si el uso de una técnica por sobre otra genera efectos favorables en las restauraciones a corto y largo plazo. Este resumen tiene como objetivo determinar cuál de estos tipos de aislación es el más efectivo en restauraciones con resina compuesta.

\section{MÉTODOS}

Realizamos una búsqueda en Epistemonikos, la mayor base de datos de revisiones sistemáticas en salud, la cual es mantenida mediante búsquedas en múltiples fuentes de información, incluyendo MEDLINE, EMBASE, Cochrane, entre otras. Extrajimos los datos desde las revisiones identificadas y analizamos los datos de los estudios primarios. Con esta información, generamos un resumen estructurado denominado FRISBEE (Friendly Summaries of Body of Evidence using Epistemonikos), siguiendo un formato preestablecido, que incluye mensajes clave, un resumen del conjunto de evidencia (presentado como matriz de evidencia en Epistemonikos), metanálisis del total de los estudios cuando sea posible, una tabla de resumen de resultados con el método GRADE y una sección de otras consideraciones para la toma de decisión.

\section{Mensajes clave}

- Realizar aislación relativa comparado con aislación absoluta podría aumentar el riesgo de falla de restauración a los 6 meses (certeza de la evidencia baja).

- No es posible establecer con claridad si la aislación relativa disminuye el riesgo de falla de la restauración a 10 años, debido a que la certeza de la evidencia existente ha sido evaluada como muy baja.

- No se encontraron estudios que evaluaran los desenlaces caries secundaria, sensibilidad postoperatoria y tinción.

Acerca del conjunto de evidencia para esta pregunta

Cuál es la evidencia

Véase matriz de evidencia en Epistemonikos más abajo.

Qué tipo de pacientes incluyeron los estudios*

Que tipo de intervenciones incluyeron los estudios*

Qué tipo de desenlaces
midieron

midieron
Encontramos tres revisiones sistemáticas ${ }^{(4,2,5)}$ que que en conjunto incluyeron dos estudios primarios ${ }^{(6,7)}$, ambos correspondientes a ensayos aleatorizados.

Ambos ensayos $^{(6,7)}$, incluyeron a pacientes con restauraciones de resina compuesta en dientes permanentes.

Un ensayo ${ }^{(6)}$ incluyó restauraciones en dientes con lesiones cervicales no cariosas en premolares inferiores, sin trauma oclusal y con pulpa vital, en dentina sin exposición pulpar y lesiones sobre margen gingival. E otro ensayo ${ }^{(7)}$ evaluó dientes con restauraciones proximales de resina en dientes permanentes.

Ambos ensayos ${ }^{(6,7)}$ compararon la realización de restauración de resina compuesta con protocolo de aislación relativa con tórulas de algodón en comparación a aislación absoluta con dique de goma.

Las revisiones sistemáticas identificadas reportaron el desenlace fracaso de la restauración, que fue evaluado por los ensayos a seis meses $^{(6)}$ y $10^{(7)}$ años de seguimiento.

* La información sobre los estudios primarios es extraída desde las revisiones sistemáticas identificadas, no directamente desde los estudios, a menos que se especifique lo contrario.

\section{RESUMEN DE LOS RESULTADOS}

La información sobre los efectos de la aislación relativa comparado con aislación absoluta en restauraciones de resina compuesta está basada en dos ensayos aleatorizados que incluyeron 262 dientes.

Un ensayo evaluó el desenlace falla de la restauración a los 6 meses (162 restauraciones $)^{(6)}$ y el otro ensayo evaluó el desenlace falla de la restauración a 10 años (100 restauraciones) $)^{(7)}$.

Los desenlaces caries secundaria, sensibilidad postoperatoria y tinción no fueron medidos o reportados por la evidencia analizada.

El resumen de los resultados es el siguiente:

- Realizar aislación relativa comparado con aislación absoluta podría aumentar el riesgo de falla de restauración a los 6 meses (certeza de la evidencia baja)

- No es posible establecer con claridad si la aislación relativa disminuye el riesgo de falla de la restauración a 10 años, debido a que la certeza de la evidencia existente ha sido evaluada como muy baja

- No se encontraron estudios que evaluaran el desenlace caries secundaria.

- No se encontraron estudios que evaluaran el desenlace sensibilidad postoperatoria

- No se encontraron estudios que evaluaran el desenlace tinción. 


\section{OTRAS CONSIDERACIONES PARA LA TOMA DE DECISIÓN}

\section{A quién se aplica y a quién no se aplica esta evidencia}

- Las conclusiones de este resumen son aplicables a pacientes con dentición permanente que requieren restauraciones de resina compuesta. Pese a que los ensayos incluidos en este resumen sólo incluyeron pacientes con restauraciones cervicales y proximales, no existen motivos para creer que los distintos tipos de aislación pudiesen funcionar diferente dependiendo de la ubicación de la restauración.

- Esta evidencia no aplica a aquellos pacientes con dentición permanente en tratamiento con aparatología de ortodoncia fija que requieran una restauración de resina, ya que en ellos no es posible realizar aislación absoluta.

\section{Sobre los desenlaces incluidos en este resumen}

- Los desenlaces incluidos son considerados críticos para la toma de decisión de acuerdo a la opinión de los autores de este resumen. Los autores consideran que la presencia de caries secundaria, sensibilidad post operatoria y tinción son desenlaces relevantes para la toma de decisiones, sin embargo no fueron medidos por las revisiones sistemáticas.

\section{Balance daño/beneficio y certeza de la evidencia}

- La evidencia indica que el uso de aislación relativa comparado con aislación absoluta podría aumentar el riesgo de falla de restauración a corto plazo (6 meses), pero la certeza de la evidencia es baja.

- Los desenlaces caries secundaria, sensibilidad y tinción no fueron reportados por la evidencia identificada.

- Considerando lo anterior, no es posible realizar un adecuado balance entre daño y beneficios. Sin embargo, la toma de decisión debe tener en cuenta la experiencia del clínico para la realización de la aislación absoluta, las características clínicas del paciente y sus preferencias.

\section{Consideraciones de recursos}

- Ninguno de las revisiones sistemáticas incluidas realizó un análisis de costos entre la aislación relativa y absoluta.

\section{Qué piensan los pacientes y sus tratantes}

- Enfrentados a la evidencia existente y debido a la incertidumbre existente, la mayoría de los pacientes y tratantes no deberían presentar preferencia por un método de aislación por sobre otro. En la práctica habitual, los tratantes tienen una inclinación hacia el uso de aislación relativa ya que técnicamente es más simple y disminuye los tiempo clínicos, pero considerando lo fundamental que es el control de la humedad durante el procedimiento clínico de restauraciones de resina compuesta, el uso de goma dique debería ser la elección, ya que no solo permite el control de la humedad, sino además separa el campo operatorio, aísla tejidos blandos, impide la deglución o aspiración de elementos.

- Además, en el contexto actual de la pandemia por COVID-19, sería una herramienta para el control del aerosol contaminante aumentando la seguridad del paciente durante la atención, entre otras ventajas ${ }^{(8)}$.

- Los pacientes podrían tener una preferencia frente al uso de la aislación relativa ya que presenta menor incomodidad en comparación al uso de la aislación absoluta.

\section{Diferencias entre este resumen y otras fuentes}

- Las conclusiones de este resumen no coinciden con las de las revisiones sistemáticas identificadas, ya que estas concluyen que no existen diferencias en la tasa de fracaso de restauraciones de resina compuesta realizadas con aislación relativa y las realizadas con aislación absoluta. Estas diferencias se deben a que las revisiones sistemáticas incluyeron ensayos que comparaban las técnicas de aislación utilizando materiales de restauración diferentes a la resina compuesta ${ }^{(4,2,5)}$.

- No se identificó ninguna guía de práctica clínica que incluyeran recomendaciones sobre el uso de un tipo de aislación por sobre otra.

\section{¿Puede que cambie esta información en el futuro?}

- La probabilidad de que futura investigación cambie las conclusiones de este resumen es alta para todos los desenlaces, debido a la incertidumbre de la evidencia existente.

- Luego de realizar una búsqueda en la International Clinical Trials Registry Platform de la Organización Mundial de la Salud y en la base de datos PROSPERO, no se identificaron registros de ensayos clínicos ni revisiones sistemáticas relacionados con la pregunta de investigación.

\section{CÓMO REALIZAMOS ESTE RESUMEN}

Mediante métodos automatizados y colaborativos recopilamos toda la evidencia relevante para la pregunta de interés y la presentamos en una matriz de evidencia.

\section{Siga el enlace para acceder a la versión interactiva:}

Aislamiento relativo comparada con aislamiento absoluto en restauraciones de resina compuesta.

\section{NOTAS}

Si con posterioridad a la publicación de este resumen se publican nuevas revisiones sistemáticas sobre este tema, en la parte superior de la matriz se mostrará un aviso de "nueva evidencia".

Este artículo es parte del proyecto síntesis de evidencia de Epistemonikos. Se elabora con una metodología preestablecida, siguiendo rigurosos estándares metodológicos y proceso de revisión por pares interno. Cada uno de estos artículos corresponde a un resumen, denominado FRISBEE (Friendly Summary of Body of Evidence using Epistemonikos), cuyo principal objetivo es sintetizar el conjunto de evidencia de una pregunta específica, en un formato amigable a los profesionales clínicos. Sus principales recursos se basan en la matriz de evidencia de Epistemonikos y análisis de resultados usando metodología GRADE. Mayores detalles de los métodos para elaborar este FRISBEE están descritos aquí: (http://dx.doi.org/10.5867/medwave.2014.06.5997)

La Fundación Epistemonikos es una organización que busca acercar la información a quienes toman decisiones en salud, mediante el uso de tecnologías. Su principal desarrollo es la base de datos Epistemonikos (www.epistemonikos.org).

\section{DECLARACIÓN DE CONFLICTOS DE INTERESES}

Los autores declaran no tener conflictos de intereses con la materia de este artículo.

\section{AGRADECIMIENTOS}

Este resumen de evidencia fue elaborado con el apoyo metodológico del Centro Evidencia UC, Facultad de Medicina, Pontificia Universidad Católica de Chile.

\section{Bibliografía}

1. Moncada G, Fernández E, Martin J, Caro MJ, Caamaño C, Mjor I, et al. Longevidad y causas de fracaso de restauraciones de amalgama y resina compuesta. Rev Dent Chile. 2007;99(3):8-16

2. Cajazeira MR, De Sabóia TM, Maia LC. Influence of the operatory field isolation technique on tooth-colored direct dental restorations. Am J Dent. 2014;27(3):155-9. 3. Gómez M, Vargas E, Pattigno B, Tirado L. Algunas consideraciones sobre el aislamiento absoluto. MEDISAN. 2017;21(10):3066-76.

4. Wang Y, Li C, Yuan H, Wong MC, Zou J, Shi Z, et al. Rubber dam isolation for restorative treatment in dental patients. Cochrane Database Syst Rev. 2016;9(9) CD009858. doi: 10.1002/14651858.CD009858.pub2

5. Brunthaler A, König F, Lucas T, Sperr W, Schedle A. Longevity of direct resin composite restorations in posterior teeth. Clin Oral Investig. 2003;7(2):63-70. 6. MaJ. Influence of rubber dam isolation on the performance of restorations for teeth wedge-shaped defects. Chinese Community Doctors. 2012;14(309):164. 7. Raskin A, Michotte-Theall B, Vreven J, Wilson NH. Clinical evaluation of a posterior composite 10-year report. J Dent. 1999;27(1):13-9.

8. Clarkson J, Ramsay C, Richards D, Robertson C, \& Aceves-Martins M; on behalf of the CoDER Working Group. Aerosol generating procedures and their mitigation in international dental guidance documents - a rapid review. 2020. Disponible en: https://oralhealth.cochrane.org/news/aerosol-generating-procedures-and-theirmitigation-internationalguidance-documents 